\title{
Atomic Row Doubling in the STM Images of Cu(014)-O Obtained with MnNi Tips
}

\author{
A. N. Chaika, ${ }^{1}$ V. N. Semenov, ${ }^{1}$ S. S. Nazin, ${ }^{1}$ and S. I. Bozhko ${ }^{1,2}$ \\ ${ }^{1}$ Institute of Solid State Physics RAS, Chernogolovka, Moscow district, 142432 Russia \\ ${ }^{2}$ NT MDT, Zelenograd, Moscow, 124460 Russia
}

S. Murphy, K. Radican, and I. V. Shvets

CRANN, School of Physics, Trinity College, Dublin 2, Ireland

(Received 19 January 2007; published 14 May 2007)

\begin{abstract}
We report on scanning tunneling microscopy studies of the $\mathrm{Cu}(014)-\mathrm{O}$ surface using $\mathrm{MnNi}$ tips. Remarkably, the results show a regular apparent doubling of surface atomic rows in the $\{110\}$ direction. A qualitative explanation of this feature based on tight binding and density functional theory calculations of the electronic structure of the tip is presented. Double imaging of the same atom by different legs of $d_{y z}$ orbital could be the reason for the observed doubling. The orientation of the orbital is determined by the O-Mn crystal field.
\end{abstract}

PACS numbers: 68.37.Ef, 71.15.Mb, 73.20.At, 73.40.Gk

Using scanning tunneling microscope (STM) tips of different materials makes it possible to obtain different information in atomically resolved images [1-3]. One of the first explanations of the atomic features in STM images of metals was given by Chen [4,5], who said that atomic resolution is most probably due to $d_{3 z^{2}-r^{2}}$ and $p_{z}$ tip states. The tunneling matrix elements for these states are proportional to the $z$ derivative of the surface atom wave function at the center of the tip apex atom [5]. Therefore, it was predicted that the best candidates for STM tips are $d$-band metals and semiconductors with $p_{z}$ dangling bonds. When tip states with angular moment component along the surface normal $m \neq 0$ dominate near $E_{F}$, enhanced but inverted corrugations are predicted [6]. A more recent coupled tip-surface system analysis [7] also demonstrated that electron states with $m \neq 0$ should give significantly larger corrugations compared to the $m=0$ states, but the relative contributions of different orbitals can show strong dependence on the tip-surface distance. The effects of the tip electronic structure were recently observed in ultrahigh resolution atomic force microscopy experiments [8,9]. Similar effects may play a significant role in dynamic STM experiments on $\mathrm{Si}(111)$ where subatomic features could be explained by scattering of electrons into double dangling bonds of the silicon tip atoms [10]. The use of dynamic STM mode for atomic orbital imaging [10] was explained by the crucial importance of having small tipsurface separations $(3-5 \AA)$, which could be achieved reproducibly without tip breaking due to lower lateral forces with an oscillating probe.

In this Letter, we present new STM data on $\mathrm{Cu}(014)-\mathrm{O}$ obtained by employing a MnNi probe in constant current mode (Fig. 1). The rationale for using tips of this material is that a Mn atom at the apex could collect electrons via the $3 d$ orbitals. We were specifically looking for unusual atomic-scale effects, e.g., multiple imaging of each atom, to address the question: Can an atomically sharp tip behave as a multiple tip with subatomic separation between the tip apexes? The surface of $\mathrm{Cu}(014)-\mathrm{O}$ was chosen as it is well understood on the atomic scale. Furthermore, it contains both metal and oxygen ions [1], which is likely to produce a strong electric crystal field that could modify the $3 d$ orbitals of a tip apex atom placed in this field.

The experiments were performed in a LAS-3000 spectrometer $\left(P<1 \times 10^{-10}\right.$ Torr $)$ equipped with a room temperature STM GPI-300. A $\mathrm{Cu}(115)$ single crystalline sample with oxygen saturated bulk [1] was cleaned from contaminants by ion $\left(\mathrm{Ar}^{+}\right)$sputtering and annealing $\left(250-400^{\circ} \mathrm{C}\right)$. The surface was then annealed at $600^{\circ} \mathrm{C}$. Oxygen out-diffused to the surface and faceting to \{014\}-type and (001) planes occurred [1]. The peak-topeak heights in Auger electron spectra give an $I_{\mathrm{O}(512)} / I_{\mathrm{Cu}(920)}$ ratio of $0.16 \pm 0.03$ corresponding to $0.5 \mathrm{ML}$ oxygen coverage [11]. The probes were fabricated from polycrystalline $\mathrm{MnNi}$ rods $\left(10 \times 0.5 \times 0.5 \mathrm{~mm}^{3}\right)$ by direct current etching in $0.5 \mathrm{M} \mathrm{HCl}$ and sharpened in ultrahigh vacuum by etching with $0.55 \mathrm{keV} \mathrm{Ar}^{+}$ions $\left(P_{\mathrm{Ar}}=5 \times 10^{-5}\right.$ Torr $)[2]$.

$X$-ray diffraction [12,13] and STM [1] show that the $\mathrm{Cu}(014)-\mathrm{O}$ surface at $0.5 \mathrm{ML}$ coverage consists of fouratom wide (001) terraces separated by [100]-oriented monatomic steps (Fig. 2). Each terrace contains four $\mathrm{Cu}[100]$ rows and two $\mathrm{O}[100]$ rows with oxygen atoms located in the first and third rows. Previous STM studies of the $\mathrm{Cu}(014)-\mathrm{O}$ surface [1] revealed a selective visualization of atomic features governed by the electronic structure of the tip and surface. Usually one, two, or three atomic rows could be distinguished within the terraces depending on the scanning parameters and the tip state. To recover the $\mathrm{Cu}(014)-\mathrm{O}$ structure in Fig. 2 one needs additional information [12,13] and the superposition of different atomically resolved STM images [1]. An example of selective atom visualization by $\mathrm{MnNi}$ probe is given in Fig. 1(a), which displays most prominently one atomic row within 

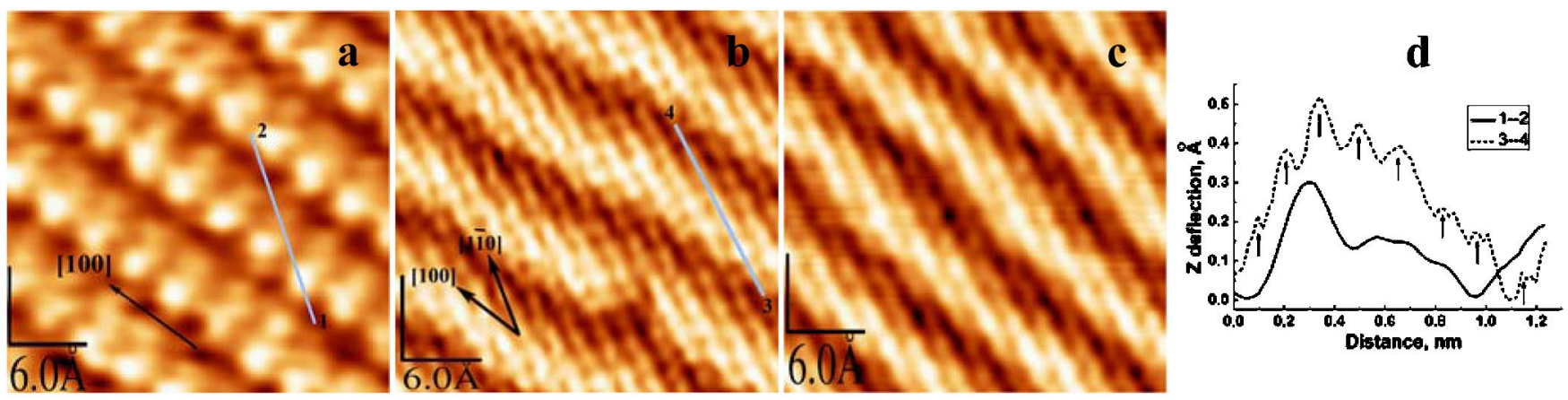

FIG. 1 (color online). (a) - (c) $3 \times 3 \mathrm{~nm}^{2} \mathrm{STM}$ images of the $\mathrm{Cu}(014)$-O surface. (a) $U=-2 \mathrm{mV}, I_{t}=0.12 \mathrm{nA}$, (b) $Y$ scan, $U=$ $-30 \mathrm{mV}, I_{t}=0.08 \mathrm{nA}$, (c) $X$ scan, $U=-30 \mathrm{mV}, I_{t}=0.08 \mathrm{nA}$. (b) and (c) were taken with different acquisition times (18 and $26 \mathrm{~s}$ ). Despite some distortion due to drift and nonorthogonality of the scanner axes, both images show doubling along [1 100$]$. A single-atom defect [Fig. 1(b)] demonstrates the sharpness of the tip. (d) Line profiles 1-2 and 3-4.

each (001) terrace while the other rows are not so prominent. Remarkably, in some cases STM images demonstrated doubling of the copper atomic features along the close-packed [110] [Figs. 1(b) and 1(c)] or [110] direction. Figures 1(b) and 1(c) display the fine structure on the 7.2 \pm $0.2 \AA$ wide terraces. We can detect 8 maxima [indicated by arrows in Fig. 1(d)] per terrace width in the line profile along [110] instead of 4 as expected from the model in Fig. 2. Meanwhile, the distance between [1 10$]$ rows agrees with the known lattice parameter. The $7.2 \AA$ step periodicity was reproduced in STM images measured at different bias voltages (from -2 to $-800 \mathrm{mV}$ ) and tunneling currents (from 0.05 to $0.8 \mathrm{nA}$ ). However, the doubling of

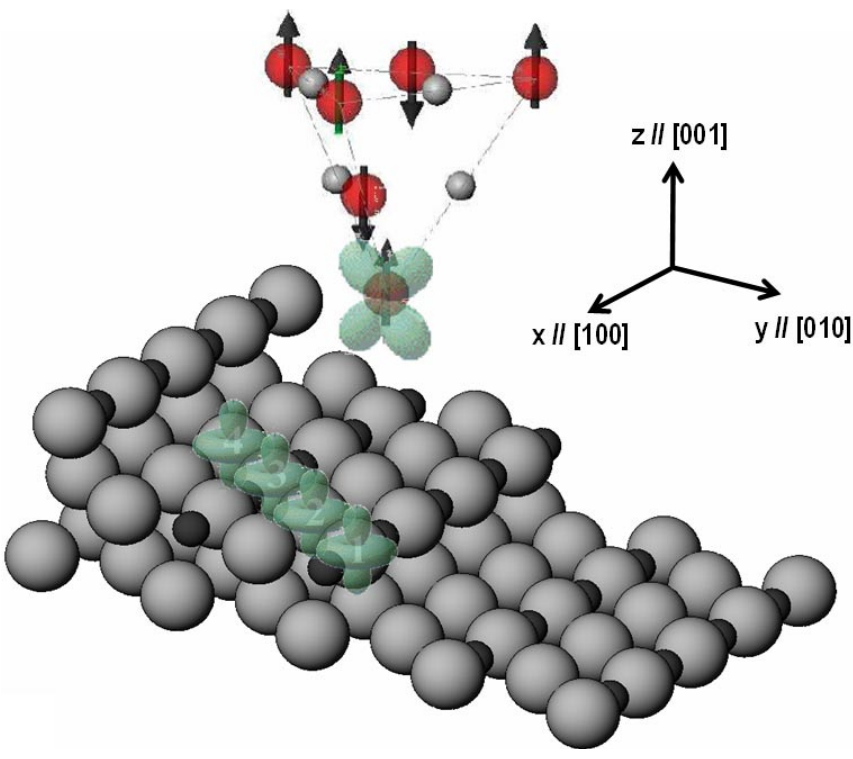

FIG. 2 (color online). Model of the $\mathrm{Cu}(014)-\mathrm{O}$ surface and MnNi tip. Large gray balls represent $\mathrm{Cu}$ atoms, small black balls denote $\mathrm{O}$, red (medium-sized) balls for $\mathrm{Mn}$, and small gray balls denote Ni. Arrows indicate the Mn magnetic moment direction. A $d_{y z}$ tip state scanning over $d_{3 z^{2}-r^{2}}$ surface states can result in doubling of the atomic features in STM images. atomic features was only observed in some experiments at sample bias voltages between -30 and $-50 \mathrm{mV}$ and currents between 0.05 and $0.08 \mathrm{nA}$. The splitting of atomic features along the [110] direction was observed less often and was not so well resolved. Typically, the images with subatomic structure appeared after a series of voltage pulses $(4-4.5 \mathrm{~V})$.

We have performed calculations of the tip electronic structure using tight-binding (TB) and density functional theory (DFT) approaches. The TB basis consisted of $d$ orbitals of the $\mathrm{Mn}$ and $\mathrm{Ni}$ atoms. At room temperature, $\mathrm{MnNi}$ has a tetragonal CuAu-I-type cell $(a=3.714 \AA$, $c=3.524 \AA$ [2]). Hopping parameters in the TB Hamiltonian accounting for the first 12 nearest neighbors were taken from the tables of Harrison [14] (the values of $r_{d}$ for $\mathrm{Mn}-\mathrm{Mn}$ and Ni-Ni hops were taken to be $r_{d}^{\mathrm{Mn}}=0.86$ and $r_{d}^{\mathrm{Ni}}=0.71 \AA$, for MnNi hops we used $r_{d}=r_{d}^{\mathrm{MnNi}}=$ $\left.\left(r_{d}^{\mathrm{Mn}} r_{d}^{\mathrm{Ni}}\right)^{1 / 2}\right)$. Although it was not crucial to our experiments, we accounted for the antiferromagnetic structure of $\mathrm{MnNi}$ by adopting spin-dependent $\mathrm{Mn}$ on-site energies $\varepsilon_{\uparrow \mathrm{Mn}}=-3 \mathrm{eV}, \varepsilon_{\downarrow \mathrm{Mn}}=-2 \mathrm{eV}$ for the two Mn sublattices (together with $\varepsilon_{\mathrm{Ni}}=-4 \mathrm{eV}$ this gives a bulk magnetic moment for Mn of $3.45 \mu_{B}$ ), as this may indirectly affect the electron density of states (DOS) through the Mn on-site energies. The tip was modeled by [001]-oriented tetrahedral and [111]-oriented trihedral clusters. The edges of the trihedral pyramid were along the diagonals of the cube faces distorted by shrinking along the $c$ axis with the coefficient $a / c=1.054$. The atom positions in the pyramids were taken to be the same as in bulk MnNi. To obtain the histogram for the partial DOS (PDOS) at the apex atom for all $d$ orbitals the energy axis was divided into $40 \mathrm{meV}$ intervals. Both tetrahedral and trihedral pyramids were analyzed with a $\mathrm{Ni}$ or $\mathrm{Mn}$ apex atom (in the latter case the on-site energies at the apex atom were chosen to favor spin-up electron occupation). Although the PDOS at the tip apex atom are still sensitive to the cluster size, the contribution of different $d$ orbitals in the vicinity of $E_{F}$ can be 


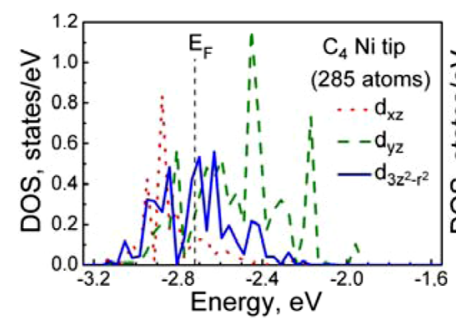

a b

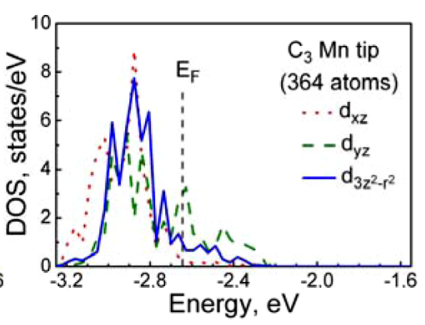

FIG. 3 (color online). (a) DOS for $d$ orbitals of a Ni atom at the tip of a [001]-oriented tetrahedral pyramid (9 layers consisting of $165 \mathrm{Ni}$ and $120 \mathrm{Mn}$ atoms). $E_{F}=-2.72 \mathrm{eV}$. (b) DOS for spinup electrons at $d$ orbitals of a $\mathrm{Mn}$ atom at the tip of a trihedral pyramid (11 layers, $182 \mathrm{Ni}$ and $182 \mathrm{Mn}$ atoms). $E_{F}=$ $-2.64 \mathrm{eV}$.

drastically different in the case of $\mathrm{Ni}$ - and $\mathrm{Mn}$-terminated tips [Figs. 3(a) and 3(b)].

Non-spin-polarized DFT calculations were performed using the plane-wave pseudopotential code CASTEP [15] with the Perdew-Wang gradient-corrected exchangecorrelation functional [16]. The tip was modeled by a five-layer $\mathrm{MnNi}(111)$ slab, with $\mathrm{Mn}$ or $\mathrm{Ni}$ atoms placed at one of the four coordination sites on the surface labeled S1-S4, identified in Fig. 4. The tip was optimized and the PDOS calculated using a $12 \AA$ vacuum gap, a plane-wave cutoff energy of $270 \mathrm{eV}$, and a $3 \times 3 \times 1 k$-point sampling grid.

The current flowing through the STM probe is governed by the electron DOS associated with the orbitals localized at the apex atom. The $x$ or $y$ axes of the orbitals can be selected by the in-plane crystal field from the sample which has different values for the [100] and [110] directions. Therefore, the observed doubling of atomic rows in one

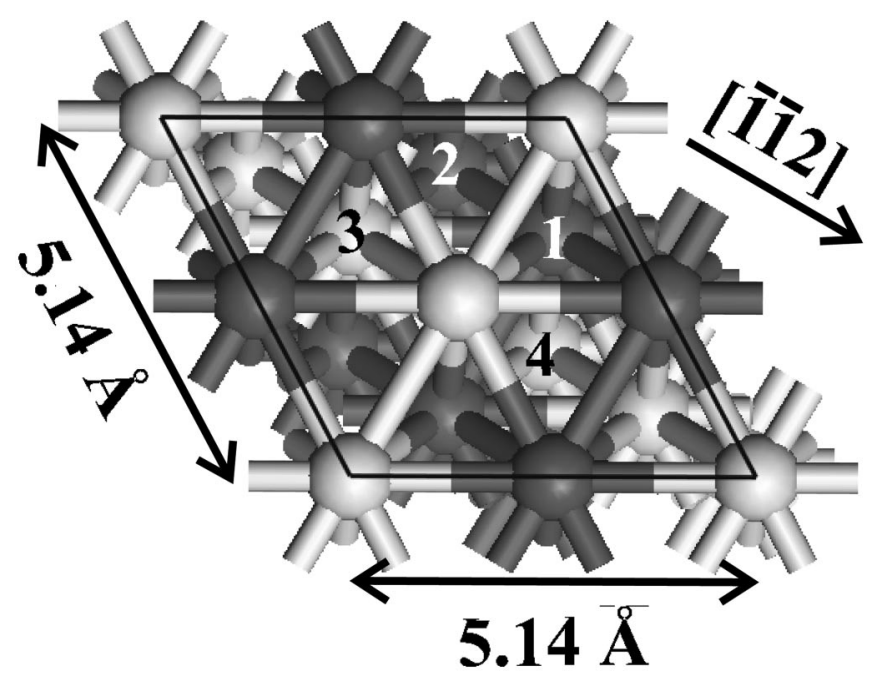

FIG. 4. Top view of the $\mathrm{MnNi}(111)$ slab, showing the four threefold coordination sites. Light gray atoms are Mn and dark gray atoms are Ni. Sites 1 and 3 are hcp sites, while sites 2 and 4 are fcc sites. of the directions can be related to a possible asymmetry of the DOS at the $d_{x z}$ and $d_{y z}$ orbitals at the apex atom. If the DOS of the $d_{y z}$ orbital substantially exceeds that of the $d_{x z}$ orbital, the butterfly-like shape of the $d_{y z}$ could result in the doubling of atomic features along the close-packed direction. A tetrahedral pyramid with a Mn apex atom possesses $C_{4 v}$ symmetry and the DOS at the $d_{x z}$ and $d_{y z}$ are identical regardless of the pyramid orientation relative to the surface. However, the situation is completely different for the three remaining pyramid types. If we consider a tetrahedral pyramid with a Ni apex, its symmetry is reduced to $C_{2 v}$ since the second layer of the pyramid consists of four $\mathrm{Mn}$ atoms positioned at the corners of a square whose diagonals feature $\mathrm{Mn}$ atoms with opposite magnetic moment. Therefore, the symmetry adapted linear combinations of $d_{x z}$ and $d_{y z}$ orbitals belonging to different irreducible representations of $C_{2 v}$ correspond to the orbitals oriented along these diagonals. Hence, $\rho_{d_{x z}}$ will only coincide with $\rho_{d_{y z}}$ if the $x$ and $y$ axes are parallel to the square sides. In this case the symmetrized orbitals yield equal contributions to both $d_{x z}$ and $d_{y z}$ orbitals. The difference between $\rho_{d_{x z}}$ and $\rho_{d_{y z}}$ becomes largest when the $x$ and $y$ axes are chosen along the square diagonals, as shown in Fig. 3(a). The most impressive situation occurs for the trihedral pyramid with the $\mathrm{Mn}$ atom at the tip where the second atomic layer is a triangle consisting of two $\mathrm{Ni}$ and one $\mathrm{Mn}$ atoms. Here, the symmetry is reduced to $C_{s}$ corresponding to reflection in the plane orthogonal to the straight line passing through the two $\mathrm{Ni}$ atoms. If that line is chosen as the $y$ axis, the $d_{x z}$ and $d_{y z}$ orbitals of the apex atom belong to different representations of $C_{s}$ and should not be expected to have equal DOS for a particular energy. Indeed, the calculations reveal that in the energy range of $\pm 50 \mathrm{meV}$ around $E_{F}$ the DOS related to the $d_{y z}$ orbital $\rho_{d_{y z}}$ strongly exceeds both $\rho_{d_{x z}}$ and $\rho_{d_{3 z^{2}-r^{2}}}$ [Fig. 3(b)]. The images presented in Figs. 1(b) and 1 (c) were taken at a small negative bias of $-30 \mathrm{meV}$, corresponding to tunneling into the tip states just above $E_{F}$, which is consistent with the dominance of $\rho_{d_{y z}}$ over $\rho_{d_{x z}}$ and $\rho_{d_{3 z^{2}-r^{2}}}$. The STM data and TB calculations are in qualitative agreement with the analysis of the tip-surface system [7], demonstrating that at small tip-surface separations both $d_{3 z^{2}-r^{2}}$ and $d_{x z}, d_{y z}$ orbitals contribute to the tunneling current. The observed atom splitting at low bias voltages [Figs. 1(b) and 1(c)] also correlates with the requirement of small tip-sample separation suggested in Ref. [10].

In the DFT calculations, we are most interested in the shape of the tip orbitals in the vicinity of $E_{F}$. Figure 5 shows orbital maps for $\mathrm{Mn}$ apex atoms in the energy interval $E_{F} \pm 0.22 \mathrm{eV}$ displayed in a vertical slice through the slab along [ [ $\left.\begin{array}{lll}1 & 1\end{array}\right]$ and horizontal slices through the apex atom and just above it. The $x, y, z$ directions (i.e., [001] direction) are all oriented out of the (111) surface. Taken together the shape of the tip orbital can be inferred. For 


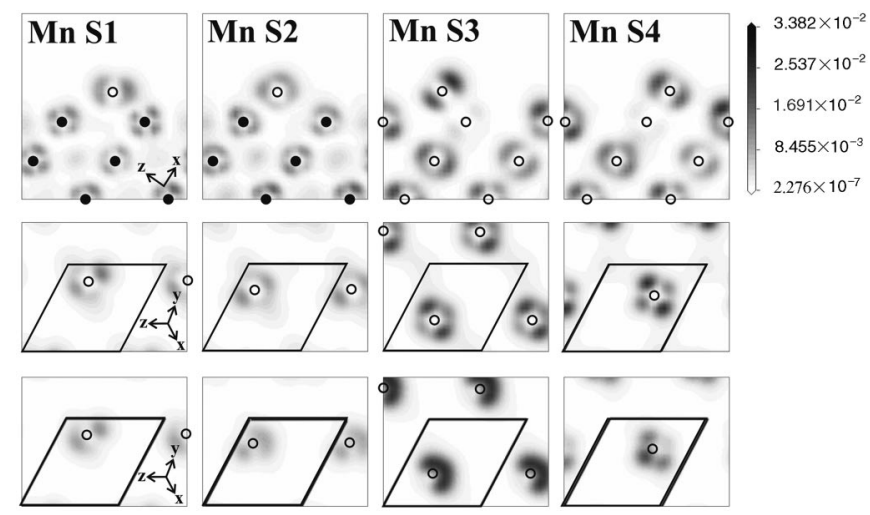

FIG. 5. Electron density (electrons $/ \AA^{3}$ ) in the energy interval $E_{F} \pm 0.22 \mathrm{eV}$ for $\mathrm{Mn}$ apex atoms at the four coordination sites (S1, S2, S3, S4) on $\mathrm{MnNi}(111)$. Upper panels display maps in a vertical cut through the slab along the $\left[\begin{array}{lll}1 & 1 & 2\end{array}\right]$ direction. Middle panels show a horizontal cut through the apex atom (slab unit cell indicated). Lower panels show a cut $0.5 \AA$ above the apex atom. Mn atoms are indicated by empty circles and Ni atoms by solid circles.

example, the MnS3 tip displays $d_{x y}$ character with four lobes lying in the $x-y$ plane, while the MnS4 tip displays $d_{x z}$ or $d_{y z}$ character. Mn or $\mathrm{Ni}$ atoms at $\mathrm{S} 3$ or $\mathrm{S} 4$ coordination sites yield the sharpest contours with a single lobe protruding into vacuum, and would therefore be expected to produce the highest resolution as STM tips. On the other hand, the MnS1 tip (i.e., a Mn apex atom located over an hcp site comprising two $\mathrm{Mn}$ and one $\mathrm{Ni}$ atom) produces twin lobes that protrude equally into the vacuum, which could be responsible for the observed atomic doubling.

The trihedral tip with a $\mathrm{Ni}$ apex atom also exhibits $\rho_{d_{x z}} \neq \rho_{d_{y z}}$, although this difference is not as spectacular. The STM image is also affected by the tip-surface interaction. The results of DFT calculations concerning the tipsample interaction (and Ni-terminated tips) will be published elsewhere; here we only mention that if the tipsurface separation becomes comparable to the interatomic distance, the interaction becomes important. We expect that the orbital orientation in our STM experiments is determined by a rather strong O-Mn crystal field adjusting the active orbital to one of the distinguished directions in the surface plane and this is the reason why the doubling was observed typically in one of the $\{110\}$ directions. The fact that the doubling of the copper atomic features is observed infrequently and under certain tunneling conditions can be explained as follows. Because of selective imaging of the surface atoms one should adjust the separation in such a way that the $d_{3 z^{2}-r^{2}}$ surface states give the largest contribution to the tunneling current and apply a bias voltage where the $d_{y z}$ orbitals dominate in the tip electronic structure (Fig. 2). The separation could not be too large (the tip $d_{y z}$ state contribution can become insufficient) nor too small (surface states with nodal symmetry can smear the atomic features). We suggest that the necessary separations lie in a rather narrow range and that the required combination of bias voltage and tip-surface distance is not easily achieved in the experiment. Moreover, detailed results of DFT calculations for 12 possible (001) and (111) oriented $\mathrm{MnNi}$ tips (to be published) demonstrate that only the MnS1 tip can be responsible for the observed asymmetry effects. This is why the success rate for observing the doubling of atomic features is only several percent.

In conclusion, we have presented new STM data on $\mathrm{Cu}(014)-\mathrm{O}$ obtained with $\mathrm{MnNi}$ probes and results of DOS calculations for $\mathrm{MnNi}$ tips with different atomic arrangements at the apex providing a qualitative explanation for the observed doubling of atomic features at the surface. The results prove that the structure of the outer shell orbitals and their partial DOS at the tip apex atom play a dominant role in the nature of atomically resolved STM image formation. Our data also highlight the importance of modification of the electronic structure of the tip by the crystal field of the sample. The results are very sensitive to the position and type of the tip apex atom. Finally, our calculations demonstrate that some kind of "multiple tip effect" may also be present even for atomically sharp tips.

This work was supported by the Russian Academy of Sciences, NT-MDT, RSSF, and FASI grants, Project No. MK-2370.2005.2, Science Foundation Ireland (No. 00/PI.1/C042), and the EU project ASPRINT No. NMP-CT-2003-001601.

[1] A. N. Chaika and S. I. Bozhko, JETP Lett. 82, 416 (2005).

[2] S. F. Ceballos et al., Surf. Sci. 523, 131 (2003).

[3] R. Wiesendanger and M.Bode, Solid State Commun. 119, 341 (2001).

[4] C. J. Chen, Phys. Rev. Lett. 65, 448 (1990).

[5] C. J. Chen, Phys. Rev. B 42, 8841 (1990).

[6] C. J. Chen, Phys. Rev. Lett. 69, 1656 (1992).

[7] W. Sacks, Phys. Rev. B 61, 7656 (2000).

[8] F. J. Giessibl et al., Science 289, 422 (2000).

[9] S. Hembacher, F. J. Giessibl, and J. Mannhart, Science 305, 380 (2004).

[10] L. A. Zotti, W. A.Hofer, and F. J. Giessibl, Chem. Phys. Lett. 420, 177 (2006).

[11] M. Wuttig, R. Franchy, and H. Ibach, Surf. Sci. 213, 103 (1989).

[12] D. A. Walko and I. K. Robinson, Surf. Rev. Lett. 6, 851 (1999).

[13] E. Vileg et al., Surf. Sci. 516, 16 (2002).

[14] W. Harrison, Electronic Structure and the Properties of Solids (Freeman, San Franscisco, 1980).

[15] M.D. Segall et al., J. Phys. Condens. Matter 14, 2717 (2002).

[16] J. P. Perdew et al., Phys. Rev. B 46, 6671 (1992). 